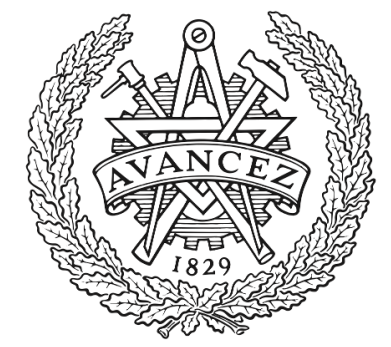

CHALMERS

UNIVERSITY OF TECHNOLOGY

\title{
5G multi-BS positioning with a single-antenna receiver
}

Downloaded from: https://research.chalmers.se, 2023-04-26 09:45 UTC

Citation for the original published paper (version of record):

Gertzell, P., Landelius, J., Nyqvist, H. et al (2020). 5G multi-BS positioning with a single-antenna receiver. IEEE International Symposium on Personal, Indoor and Mobile Radio Communications, PIMRC, 2020-August. http://dx.doi.org/10.1109/PIMRC48278.2020.9217124

N.B. When citing this work, cite the original published paper. 


\title{
5G multi-BS Positioning with a Single-Antenna Receiver
}

\author{
Philip Gertzell ${ }^{* \S}$, Jacob Landelius*§, Hanna Nyqvist ${ }^{\S}$, Alessio Fascista ${ }^{\ddagger}$, Angelo Coluccia ${ }^{\ddagger}$, \\ Gonzalo Seco-Granados ${ }^{\dagger}$, Nil Garcia*, Henk Wymeersch* \\ *Department of Electrical Engineering, Chalmers University of Technology, Sweden, ${ }^{\S}$ Aptiv, Sweden \\ ${ }^{\ddagger}$ Department of Innovation Engineering, University of Salento, Lecce, Italy \\ ${ }^{\dagger}$ Department of Telecommunications and Systems Engineering, Universitat Autonoma de Barcelona, Spain
}

\begin{abstract}
Cellular localization generally relies on timedifference-of-arrival (TDOA) measurements. In this paper, we investigate a novel scenario where the mobile user estimates its own position by jointly exploiting TDOA and angle of departure (AOD) measurements, which are estimated from downlink transmissions in a millimeter-wave (mmWave) multiple-input singleoutput (MISO) setup. We first perform a Fisher information analysis to derive the lower bounds on the estimation accuracy, and then propose a novel localization algorithm, which is able to provide improved performance also with few transmit antennas and limited bandwidth.
\end{abstract}

\section{INTRODUCTION}

Localization in cellular systems has received significant attention in the last decades, and nowadays it is considered as a key requirement for most applications in the emerging $5 \mathrm{G}$ networks [1].

Localization in 4G systems generally relies on TDOA measurements in either uplink (UL) or downlink (DL) channel [2], [3]. In uplink TDOA (U-TDOA) localization, a mobile station (MS) with unknown position transmits a signal and a set of base stations (BSs), placed at known locations and perfectly synchronized among each other, record the time of arrival (TOA) of such a signal. Then, the MS position is estimated by processing the time differences among the TOA measurements collected at the BSs. Downlink TDOA (O-TDOA) uses the same position estimation principle as in U-TDOA, but the time differences are computed based on the TOAs measured at the MS.

Different techniques have been proposed in the literature to estimate the MS position from a set of TDOA measurements: for instance, in [4] authors proposed a closed-form solution to the hyperbolic positioning problem that traces out the possible MS locations. The adoption of iterative algorithms has also been considered to lower the complexity involved in the TDOAbased position estimation process [5], [6]. From release 9, the LTE standard included a set of positioning reference signals, which have been largely exploited to devise more accurate localization approaches [7], [8].

A further enhancement can be obtained by combining TDOA measurements with angular information, which represent a very useful source of information for positioning. The most direct

Philip Gertzell and Jacob Landelius contributed equally to this work. way to obtain angular measurements is using angle of arrival (AOA); however, since antenna arrays are typically available at the BSs side, this implies a frequent use of the UL channel. Authors in [9] proposed a two-step least squares (LS) estimator based on hybrid TDOA/AOA information, which is able to attain much higher accuracy than TDOA-only. The same finding has been confirmed in [10], where it is shown that the combined use of TDOA/AOA enables MS positioning with only two BSs, rather than the three required in TDOA-only systems.

The advent of the $5 \mathrm{G}$ technology is opening the doors to unprecedented opportunities, including novel features that will dramatically improve the positioning capabilities of future cellular networks. Indeed, it has been shown that the use of mmWave and the adoption of a massive number of antennas at both transmit and receive side (MIMO) can provide accuracy below one meter [11], [12]. However, in the near future it is expected that a large number of antennas will be available only on the BS side, i.e., a MISO setup. Given the highly directional nature of $5 \mathrm{G}$ communications, an interesting alternative to perform angular measurements consists in estimating the AOD of the transmitted signals. This has two main advantages: first, the positioning process can be carried out at the MS by exploiting DL broadcast transmissions, resulting in a significant saving of UL resources. Second, it enables localization of MIMO devices even in presence of severe obstructions, if just one antenna is in line-of-sight (LOS) with a BS [13].

In this paper, we address the problem of estimating the MS position based on the combined use of TDOA and AOD measurements, using DL-only transmissions under a mmWave MISO setup. First, we carry out a Fisher information analysis aimed at providing a theoretical assessment of the achievable performance, also taking into account the presence of a synchronization bias at the MS. As a second contribution, we propose a two-step algorithm, which provides improved localization performance even with few transmit antennas and lower bandwidth.

\section{SySTEM MODEL}

We consider a MISO-OFDM system having bandwidth $W_{\mathrm{B}}=1 / T_{s}$, with $N_{\mathrm{BS}}$ perfectly synchronized BSs each equipped with $N_{t}$ antennas. The two-dimensional position of each BS $i$ is known and denoted $\mathbf{p}_{i}=\left[\begin{array}{ll}x_{i} & y_{i}\end{array}\right]^{\mathrm{T}} \in \mathbb{R}^{2}$. 
The MS is limited to one antenna and has unknown position $\mathbf{p}=\left[\begin{array}{ll}p_{x} & p_{y}\end{array}\right]^{\mathrm{T}}$ and unknown clock bias $b$. Each BS sends $G$ consecutive OFDM symbols, where the pilot on subcarrier $n \in\{0, \ldots, N-1\}$ during OFDM symbol $g \in\{0, \ldots, G-1\}$ is denoted by $\mathbf{s}_{i}^{g}[n] \in \mathbb{C}^{N_{t} \times 1}$. Note that any precoding at the $\mathrm{BS}$ is already subsumed in these symbols. The transmitted pilot signals are considered orthogonal between BSs.

\section{A. Channel Model}

We assumed that a pure LOS path, without reflections, is present from each BS to the MS. An overview of the setup is shown in Fig. 1 with the relevant channel parameters. We assume a stationary channel during all $G$ transmissions and the complex channel (row) vector on subcarrier $n$ from $\mathrm{BS} i$ to the MS is $\mathbf{h}_{i}^{\mathrm{T}}[n]=\gamma_{i}[n] \mathbf{a}_{i}^{\mathrm{H}}\left(\theta_{i}\right) \in \mathbb{C}^{N_{t}}, i=1, \ldots, N_{\mathrm{BS}}$, where $\mathbf{a}_{i}^{\mathrm{H}}\left(\theta_{i}\right)$ is the antenna response vector of BS $i$ and $\gamma_{i}[n]$ the complex channel gain on subcarrier $n$, modeled as $\gamma_{i}[n]=$ $\alpha_{i} \exp \left(-j 2 \pi n \tau_{i} /\left(N T_{s}\right)\right)$, where $\tau_{i}=\left\|\mathbf{p}-\mathbf{p}_{i}\right\| / c+b$ is the measured TOA, $\alpha_{i}=\rho_{i} e^{j \phi_{i}}$ captures the path loss with $\rho_{i}=$ $\lambda_{c} /\left(4 \pi d_{i}\right)$, with $\phi_{i}$ as a random uniformed distributed variable between $[0,2 \pi]$ and $d_{i}=\left\|\mathbf{p}-\mathbf{p}_{i}\right\|$ is the distance from a BS to the MS, while $\lambda_{c}$ is the wavelength at the carrier frequency when assuming narrowband characteristics.

The antenna response vector is assumed to be an ULA with $\lambda_{c} / 2$ spaced isotropic antennas, so that

$$
\left[\mathbf{a}_{i}\left(\theta_{i}\right)\right]_{n}=e^{-j n \pi \sin \left(\theta_{i}\right)}, n=0,1, \ldots, N_{t}-1,
$$

where $\theta_{i}=\operatorname{atan} 2\left(p_{y}-y_{i}, p_{x}-x_{i}\right)+\Theta_{i}$ is the AOD from the corresponding BS with $\Theta_{i}$ being the orientation of the BS and atan $2(y, x)$ is the four-quadrant inverse tangent.

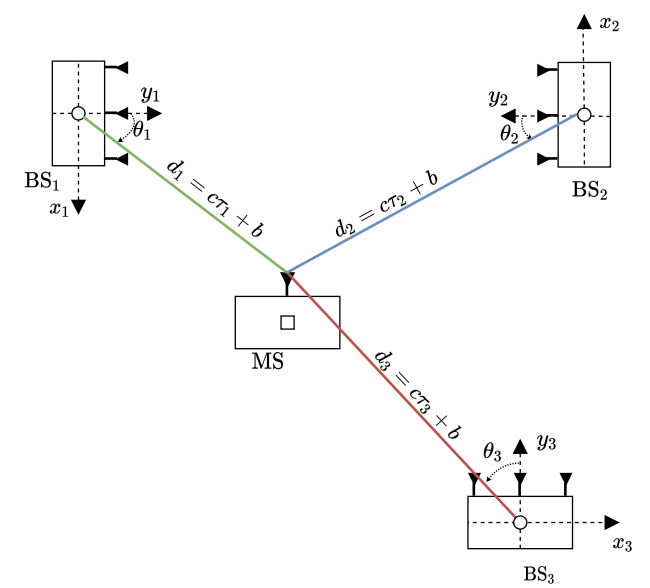

Fig. 1. Geometry of the considered scenario comprising one mobile station and several synchronized base stations.

\section{B. Receiver Model}

The received signal at the $g$-th transmission with subcarrier $n$ is modeled as

$$
y_{i}^{g}[n]=\sqrt{P_{t}} \mathbf{h}_{i}^{\mathrm{T}}[n] \mathbf{s}_{i}^{g}[n]+\omega_{i}^{g}[n]
$$

where $\omega_{i}^{g}[n]$ is additive white complex Gaussian noise with zero mean and the variance $\sigma^{2}$. The transmitter power for each antenna is denoted $P_{t}$. The observations from BS $i$ are aggregated into the matrix

$$
\mathbf{Y}_{i}=\left[\begin{array}{ccc}
y_{i}^{0}[0] & \cdots & y_{i}^{G-1}[0] \\
\vdots & \ddots & \vdots \\
y_{i}^{0}[N-1] & \cdots & y_{i}^{G-1}[N-1]
\end{array}\right]
$$

Note that the $g$-th column of $\mathbf{Y}_{i}$ can be expressed as

$$
\mathbf{y}_{i}^{g}=\sqrt{P_{t}} \alpha_{i} \tilde{\gamma}\left(\tau_{i}\right) \odot\left(\mathbf{a}_{i}^{\mathrm{H}}\left(\theta_{i}\right) \mathbf{S}_{i}^{g}\right)^{\mathrm{T}}+\boldsymbol{\omega}_{i}^{g},
$$

where $\left[\tilde{\gamma}\left(\tau_{i}\right)\right]_{n}=\exp \left(-j 2 \pi n \tau_{i} /\left(N T_{s}\right)\right)$, for $n=0, \ldots, N-1$, and $\mathbf{S}_{i}^{g}=\left[\boldsymbol{s}_{i}^{g}[0] \ldots \boldsymbol{s}_{i}^{g}[N-1]\right] \in \mathbb{C}^{N_{t} \times N}$.

\section{FISHER INFORMATION ANALYSIS}

In this section we derive the Fisher information matrix (FIM) and Cramér-Rao lower bound (CRLB) for the channel parameters and the MS location.

\section{A. FIM Derivation for Channel Parameters}

The noise-free observation for the $g$-th transmission over the $n$-th subcarrier is denoted by

$$
m_{i}^{g}[n]=\sqrt{P_{t}} \alpha_{i} e^{-j \frac{2 \pi n \tau_{i}}{N T_{s}}} \mathbf{a}_{i}^{\mathrm{H}}\left(\theta_{i}\right) \mathbf{s}_{i}^{g}[n] .
$$

The unknown channel parameter vector is $\boldsymbol{\zeta}_{i}=\left[\rho_{i}, \phi_{i}, \tau_{i}, \theta_{i}\right]^{\mathrm{T}}$. Considering $\hat{\zeta}_{i}$ as an unbiased estimator of $\boldsymbol{\zeta}_{i}$ the expression of the FIM for the channel parameters is well-known to be related to the estimation error covariance matrix as $\mathbb{E}\left[\left(\boldsymbol{\zeta}_{i}-\hat{\boldsymbol{\zeta}}_{i}\right)\left(\boldsymbol{\zeta}_{i}-\hat{\boldsymbol{\zeta}}_{i}\right)^{\mathrm{T}}\right] \succeq \mathbf{I}_{\boldsymbol{\zeta}_{i}}^{-1}[14]$, where the operator $\mathbb{E}[\cdot]$ denotes the expected value and $\mathbf{I}_{\boldsymbol{\zeta}_{i}}$ is the FIM for the channel parameters, with

$$
\mathbf{I}_{\boldsymbol{\zeta}_{i}}=\frac{2}{\sigma^{2}} \sum_{g=0}^{G-1} \sum_{n=0}^{N-1} \Re\left\{\nabla_{\boldsymbol{\zeta}}^{\mathrm{H}} m_{i}^{g}[n] \nabla_{\boldsymbol{\zeta}} m_{i}^{g}[n]\right\}
$$

where $\Re\{\cdot\}$ denotes the real-part operator and

$$
\nabla_{\zeta} m_{i}^{g}[n]=e^{j\left(\phi_{i}-\frac{2 \pi n \tau_{i}}{N T_{s}}\right)}\left[\begin{array}{c}
\mathbf{a}_{i}^{\mathrm{H}}\left(\theta_{i}\right) \mathbf{s}_{i}^{g}[n] \\
j \rho_{i} \mathbf{a}_{i}^{\mathrm{H}}\left(\theta_{i}\right) \mathbf{s}_{i}^{g}[n] \\
\frac{-j 2 \pi n}{N T_{s}} \rho_{i} \mathbf{a}_{i}^{\mathrm{H}}\left(\theta_{i}\right) \mathbf{s}_{i}^{g}[n] \\
-j \pi \cos \theta_{i} \rho_{i} \mathbf{a}_{i}^{\mathrm{H}}\left(\theta_{i}\right) \mathbf{D} \mathbf{s}_{i}^{g}[n]
\end{array}\right]
$$

where $\mathbf{D}=\operatorname{diag}\left[0, \ldots, N_{t}-1\right]$. The resulting $4 \times 4$ matrix $\mathbf{I}_{\boldsymbol{\zeta}_{i}}$ is full rank when at least two subcarriers and one transmission or at least two transmissions and one subcarrier are used with unique pilot signals [13].

\section{B. FIM Derivation for Position}

From $\mathbf{I}_{\boldsymbol{\zeta}_{i}}$ we apply a Jacobian transformation to obtain the FIM of $\boldsymbol{\eta}_{i}=\left[\rho_{i}, \phi_{i}, \mathbf{p}^{\mathrm{T}}, b\right]^{\mathrm{T}}$

$$
\mathbf{I}_{\boldsymbol{\eta}_{i}}=\mathbf{T}_{i}^{\mathrm{T}} \mathbf{I}_{\boldsymbol{\zeta}_{i}} \mathbf{T}_{i}
$$


where

$$
\mathbf{T}_{i} \stackrel{\text { def }}{=} \frac{\partial \boldsymbol{\zeta}_{i}^{\mathrm{T}}}{\partial \boldsymbol{\eta}_{i}}=\left[\begin{array}{cccc}
1 & 0 & 0 & 0 \\
0 & 1 & 0 & 0 \\
0 & 0 & \partial \tau_{i} / \partial \mathbf{p} & \partial \tau_{i} / \partial b \\
0 & 0 & \partial \theta_{i} / \partial \mathbf{p} & \partial \theta_{i} / \partial b
\end{array}\right] .
$$

The derivatives turn out to be

$$
\begin{aligned}
& \partial \tau_{i} / \partial \mathbf{p}=\frac{1}{c}\left[\cos \left(\theta_{i}\right), \sin \left(\theta_{i}\right)\right]^{\mathrm{T}} \\
& \partial \theta_{i} / \partial \mathbf{p}=\frac{1}{\left\|\mathbf{p}-\mathbf{p}_{i}\right\|}\left[-\sin \left(\theta_{i}\right), \cos \left(\theta_{i}\right)\right]^{\mathrm{T}} \\
& \partial \theta_{i} / \partial b=0, \quad \partial \tau_{i} / \partial b=1 .
\end{aligned}
$$

The result of the transformation leads to a $5 \times 5$ matrix $\mathbf{I}_{\boldsymbol{\eta}_{i}}$. This matrix is always singular, due to the fact that the clock bias is unknown. In order to deal with the singularity, the individual FIM for all BSs are combined into a collective FIM. It will be convenient to break up $\mathbf{I}_{\boldsymbol{\eta}_{i}}$ as

$$
\mathbf{I}_{\boldsymbol{\eta}_{i}}=\left[\begin{array}{ll}
\mathbf{A}_{i} & \mathbf{B}_{i} \\
\mathbf{B}_{i}^{\mathrm{T}} & \mathbf{C}_{i}
\end{array}\right]
$$

where $\mathbf{A}_{i} \in \mathbb{R}^{2 \times 2}, \mathbf{B}_{i} \in \mathbb{R}^{2 \times 3}$, and $\mathbf{C}_{i} \in \mathbb{R}^{3 \times 3}$. The FIM of the vector $\boldsymbol{\eta}$ of length $2 N_{\mathrm{BS}}+3$, with $\boldsymbol{\eta}=$ $\left[\rho_{1}, \phi_{1}, \ldots, \rho_{N_{\mathrm{BS}}}, \phi_{N_{\mathrm{BS}}}, \mathbf{p}^{\mathrm{T}}, b\right]^{\mathrm{T}}$ is of the form

$$
\mathbf{I}_{\boldsymbol{\eta}}=\left[\begin{array}{cccc}
\mathbf{A}_{1} & & & \mathbf{B}_{1} \\
& \ddots & & \vdots \\
& & \mathbf{A}_{N_{\mathrm{BS}}} & \mathbf{B}_{N_{\mathrm{BS}}} \\
\mathbf{B}_{1}^{\mathrm{T}} & \ldots & \mathbf{B}_{N_{\mathrm{BS}}}^{\mathrm{T}} & \sum_{i=1}^{N_{\mathrm{BS}}} \mathbf{C}_{i}
\end{array}\right],
$$

in which empty entries are equal to zero. The equivalent Fisher information matrix (EFIM) of the parameter $\boldsymbol{\pi}=\left[\mathbf{p}^{\mathrm{T}}, b\right]^{\mathrm{T}}$ is readily found to be

$$
\mathbf{I}_{\boldsymbol{\pi}}^{\mathrm{E}}=\sum_{i=1}^{N_{\mathrm{BS}}} \mathbf{C}_{i}-\sum_{i=1}^{N_{\mathrm{BS}}} \mathbf{B}_{i}^{\mathrm{T}} \mathbf{A}_{i}^{-1} \mathbf{B}_{i} .
$$

Finally, the position error bound (PEB) can be derived from the diagonal entries of the inverse of (15) expressed as

$$
\mathrm{PEB}=\sqrt{\operatorname{tr}\left\{\left[\mathbf{I}_{\boldsymbol{\pi}}^{\mathrm{E}}\right]_{1: 2,1: 2}^{-1}\right\}},
$$

where $\operatorname{tr}\{\cdot\}$ denotes the matrix trace.

\section{Local and Global Processing Methods}

In this section, we derive the local algorithm to estimate the AOD and TOA from each BS, and then combine these local estimates to obtain the user's position.

\section{A. Per BS Channel Estimation}

In order to estimate TOA $\tau_{i}$ and AOD $\theta_{i}$ we propose a maximum likelihood (ML) solution, as it tends to outperform methods based on harmonic retrieval, such as beamspace ESPRIT [15]. We first make use of the fact that the received signal $\mathbf{y}_{i}^{g} \in \mathbb{C}^{N \times 1}$ can be modeled as a complex Gaussian distribution such that

$$
\mathbf{y}_{i}^{g} \sim \mathcal{C N}\left(\alpha_{i} \tilde{\mathbf{h}}_{i}^{g}\left(\tau_{i}, \theta_{i}\right), \sigma^{2}\right),
$$

with $\tilde{\mathbf{h}}_{i}^{g}\left(\tau_{i}, \theta_{i}\right)$ based on (4) is defined

$$
\tilde{\mathbf{h}}_{i}^{g}\left(\tau_{i}, \theta_{i}\right)=\tilde{\gamma}\left(\tau_{i}\right) \odot\left(\mathbf{a}_{i}^{\mathrm{H}}\left(\theta_{i}\right) \mathbf{S}_{i}^{g}\right)^{\mathrm{T}} .
$$

An estimate of $\tau_{i}$ and $\theta_{i}$ is found from the ML estimator expressed as

$$
\left(\hat{\tau}_{i}, \hat{\theta}_{i}\right)=\arg \max _{\left(\tau_{i}, \theta_{i}\right)}\left[\max _{\left(\alpha_{i}, \sigma^{2}\right)} \log p\left(\mathbf{Y}_{i} \mid \tau_{i}, \theta_{i}, \alpha_{i}, \sigma^{2}\right)\right] .
$$

Exploiting the conditional independence of $\mathbf{y}_{i}^{g}$ and the structure of the Gaussian density, we find that

$$
\log p\left(\mathbf{Y}_{i} \mid \tau_{i}, \theta_{i}, \alpha_{i}\right) \propto-\sum_{g=0}^{G-1}\left\|\mathbf{y}_{i}^{g}-\alpha_{i} \tilde{\mathbf{h}}_{i}^{g}\left(\tau_{i}, \theta_{i}\right)\right\|^{2} .
$$

Solving for $\alpha_{i}$ leads to

$$
\hat{\alpha}_{i}\left(\tau_{i}, \theta_{i}\right)=\frac{\sum_{g=1}^{G}\left(\tilde{\mathbf{h}}_{i}^{g}\left(\tau_{i}, \theta_{i}\right)\right)^{\mathrm{H}} \mathbf{y}_{i}^{g}}{\sum_{g=1}^{G}\left\|\tilde{\mathbf{h}}_{i}^{g}\left(\tau_{i}, \theta_{i}\right)\right\|^{2}} .
$$

Finally the ML estimator is compressed to a matched filter

$$
\begin{aligned}
\left(\hat{\tau}_{i}, \hat{\theta}_{i}\right) & =\arg \min _{\left(\tau_{i}, \theta_{i}\right)} \sum_{g=0}^{G-1}\left\|\mathbf{y}_{i}^{g}-\hat{\alpha}_{i}\left(\tau_{i}, \theta_{i}\right) \tilde{\mathbf{h}}_{i}^{g}\left(\tau_{i}, \theta_{i}\right)\right\|^{2} \\
& =\arg \max _{\left(\tau_{i}, \theta_{i}\right)} \frac{\left|\sum_{g=1}^{G}\left(\tilde{\mathbf{h}}_{i}^{g}\left(\tau_{i}, \theta_{i}\right)\right)^{\mathrm{H}} \mathbf{y}_{i}^{g}\right|^{2}}{\sum_{g=1}^{G}\left\|\tilde{\mathbf{h}}_{i}^{g}\left(\tau_{i}, \theta_{i}\right)\right\|^{2}} .
\end{aligned}
$$

In order to solve this problem, a two-dimensional grid search is implemented, with iterative grid refinement up to a resolution determined by the CRLB.

\section{B. Global Position Estimation}

In order to remove the unknown clock bias $b$ we post-process the TOA estimates and calculate the TDOA as

$$
\Delta \hat{\tau}_{i}=\hat{\tau}_{i}-\hat{\tau}_{N_{\mathrm{BS}}}, i \neq N_{\mathrm{BS}}
$$

where we have chosen BS $i=N_{\mathrm{BS}}$ as the reference. Note that the noise-free TDOA is $\Delta \tau_{i}=\left(d_{i}-d_{N_{\mathrm{BS}}}\right) / c$, which does not depend on $b$. We thus construct an observation

$$
\hat{\boldsymbol{\psi}}=\left[\Delta \hat{\tau}_{1}, \ldots, \Delta \hat{\tau}_{N_{\mathrm{BS}}-1}, \hat{\theta}_{1}, \ldots, \hat{\theta}_{N_{\mathrm{BS}}}\right]^{\mathrm{T}} .
$$

The true TDOA and AOD, defined as $\psi$ are geometric functions of $\mathbf{p}$, such that $\boldsymbol{\psi}=f(\mathbf{p}) \in \mathbb{R}^{\left(2 N_{\mathrm{BS}}-1\right) \times 1}$ [13], where

$$
f(\mathbf{p})=\left[\begin{array}{c}
\frac{1}{c}\left(\left\|\mathbf{p}-\mathbf{p}_{1}\right\|-\left\|\mathbf{p}-\mathbf{p}_{N_{\mathrm{BS}}}\right\|\right) \\
\vdots \\
\frac{1}{c}\left(\left\|\mathbf{p}-\mathbf{p}_{N_{\mathrm{BS}}-1}\right\|-\left\|\mathbf{p}-\mathbf{p}_{N_{\mathrm{BS}}}\right\|\right) \\
\operatorname{atan} 2\left(p_{y}-y_{1}, p_{x}-x_{1}\right)+\Theta_{1} \\
\vdots \\
\operatorname{atan} 2\left(p_{y}-y_{N_{\mathrm{BS}}}, p_{x}-x_{N_{\mathrm{BS}}}\right)+\Theta_{N_{\mathrm{BS}}}
\end{array}\right] .
$$


This then immediately leads to the following weighted LS problem:

$$
\hat{\mathbf{p}}=\arg \min _{\mathbf{p}}(\hat{\boldsymbol{\psi}}-f(\mathbf{p}))^{\mathrm{T}} \boldsymbol{\Sigma}^{-1}(\hat{\boldsymbol{\psi}}-f(\mathbf{p})) .
$$

Matrix $\boldsymbol{\Sigma}^{-1}$ is the FIM of the parameters in (25), which can be obtained by a simple combination and arrangement of the elements of matrices $\mathbf{I}_{\zeta_{i}}$ for $i=1, \ldots, N_{\mathrm{BS}}$.

The two-dimensional search is implemented by first using the AOD estimations to limit the search area by finding the intersections of the $N_{\mathrm{BS}}$ bearing lines. The resolution of the search is determined by the PEB.

Remark 1. In case only the TOAs $\hat{\tau}_{i}$ are available, the problem (27) can be solved, provided $N_{\mathrm{BS}} \geq 3$. In case only the AODs $\hat{\theta}_{i}$ are available, (27) can be solved provided $N_{\mathrm{BS}} \geq 2$.

\section{Numerical Results}

In this section the estimation results are presented to evaluate the performance of the estimators in comparison to the theoretical bounds derived from the FIM.

\section{A. Simulation Setup}

As reference scenario we consider a simple traffic localization scenario, the distance from the MS to all BS are set constant to approximately $30 \mathrm{~m}$. Each BS's orientation is known $\Theta_{1}=0 \mathrm{rad}, \Theta_{2}=-\pi / 2 \mathrm{rad}$ and $\Theta_{3}=\pi \mathrm{rad}$. The BS positions are at known positions $\mathbf{p}_{1}=[-25,-17]^{\mathrm{T}} \mathrm{m}, \mathbf{p}_{2}=[17,-25]^{\mathrm{T}}$ $\mathrm{m}$ and $\mathbf{p}_{3}=[25,17]^{\mathrm{T}} \mathrm{m}$ of the considered Cartesian reference system. The MS position is unknown and located at $\mathbf{p}=[0,0]^{\mathrm{T}}$ $\mathrm{m}$. All three BSs are equipped with $N_{t} \leq 80$ antennas without any beamforming.

Three signals per BS are sequentially transmitted $G=3$ and each with a carrier frequency of $f_{c}=24 \mathrm{GHz}$, available bandwidth $W_{\mathrm{B}} \leq 700 \mathrm{MHz}$, with sampling time $T_{s}=1 / W_{\mathrm{B}}$, subcarriers $N=40$ and a clock bias of $b=20$ ns. The transmitted power per BS will be varied such that SNR range from $-10 \mathrm{~dB}$ to $15 \mathrm{~dB}$. The SNR is defined in $\mathrm{dB}$ as

$$
\mathrm{SNR}_{i}=10 \log _{10}\left(\frac{N_{t} P_{t}\left|\alpha_{i}\right|^{2}}{N_{0} W_{\mathrm{B}}}\right),
$$

where $N_{0} W_{\mathrm{B}}=T_{0} k_{\mathrm{B}} W_{\mathrm{B}}$ is the noise variance of the received signal, $k_{\mathrm{B}}$ is the Boltzmann constant, $T_{0}$ is the standard thermal noise, $\alpha_{i}$ is defined in Sec. II. To measure the performance of the estimator the metric Root Mean Squared Error (RMSE) is used and evaluated in relation to the theoretical PEB of the corresponding case. Moreover, the estimator is put in relation to estimators considering only TDOA measurements and only AOD measurements to evaluate the accuracy when a combination is used. For each simulation point, 500 Monte Carlo simulations was performed.

\section{B. Channel and Position estimation for varying SNR}

In this section we present the simulation results of the proposed scenario with varying SNR from $-10 \mathrm{~dB}$ to $15 \mathrm{~dB}$, the bandwidth is fixed to $W_{\mathrm{B}}=100 \mathrm{MHz}$ and the number of antennas $N_{t}=40$. In Fig. 2 the RMSE of the channel estimations are presented together with their corresponding theoretical bounds as $\sqrt{\mathrm{CRLB}}$. The CRLB is the inverse of the channel FIM $\boldsymbol{I}_{\boldsymbol{\zeta}_{i}}$ from (6) of the respective parameter. As seen in the figure the estimations successfully achieve the CRLB from $-5 \mathrm{~dB}$ and up. In Fig. 3 the RMSE of position estimates are plotted together with the corresponding PEB, over different SNR values. We see that $\mathrm{cm}$-level precision is possible in all cases with SNR values above $5 \mathrm{~dB}$. The proposed estimator shows overall greater accuracy than the individual estimators considering only TDOA or AOD.
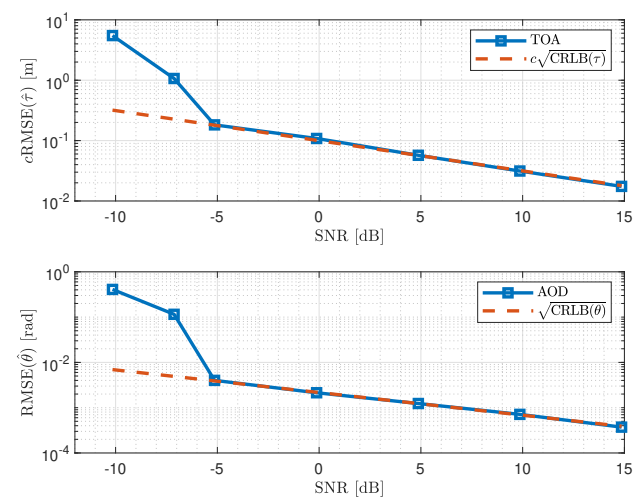

Fig. 2. RMSEs of the estimated channel parameters $d=c \tau$ and $\theta$ versus the $\sqrt{\mathrm{CRLB}}$ for the two parameters as a function of SNR for $N_{t}=40$ and $W_{\mathrm{B}}=100 \mathrm{MHz}$

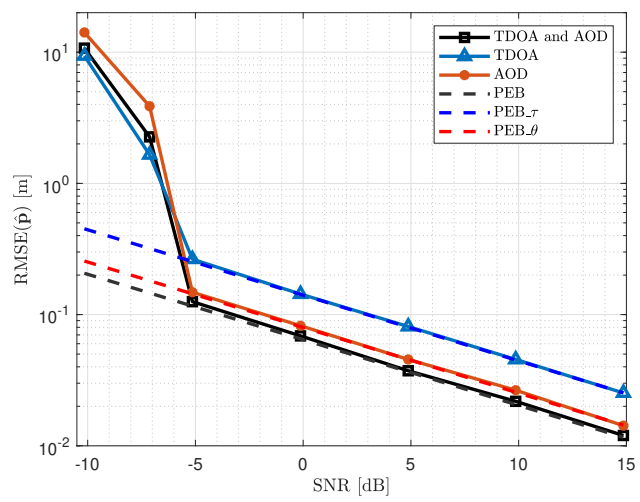

Fig. 3. RMSEs of the position estimates versus the PEB for the different estimates as a function of SNR for $N_{t}=40$ and $W_{\mathrm{B}}=100 \mathrm{MHz}$.

\section{Position estimation for varying transmit antennas}

In this scenario we consider a scenario similar as in Sec. V-B but now we variate the number of transmit antennas from 2 to 80 (for 1 , only TDOA is available) to investigate localization accuracy with few antenna elements but sufficient bandwidth. The SNR is set to $5 \mathrm{~dB}$ and bandwidth $W_{\mathrm{B}}=100 \mathrm{MHz}$. The result of the position estimations is observed in Fig. 4. With few antennas, we see a significant decrease in position estimation accuracy when only considering AOD measurements, which is to be expected. However, time measurements are eminent to 
provide accurate position estimates $<10 \mathrm{~cm}$ with very few antennas present.

\section{Position estimation for varying bandwidth}

By ranging the bandwidth from $10 \mathrm{MHz}$ to $700 \mathrm{MHz}$ the estimator performance is evaluated. SNR is set to $5 \mathrm{~dB}$ and each BS is equipped with $N_{t}=40$ antennas. As seen in Fig. 5, time measurements are inadequate in order to bring the position accuracy below $10 \mathrm{~cm}$ when the bandwidth is less than $100 \mathrm{MHz}$. However, as many antennas are utilized AOD measurements alone achieve great positioning accuracy. The proposed estimator outperforms both TDOA-only and AODonly for bandwidths between 100 and $500 \mathrm{MHz}$.

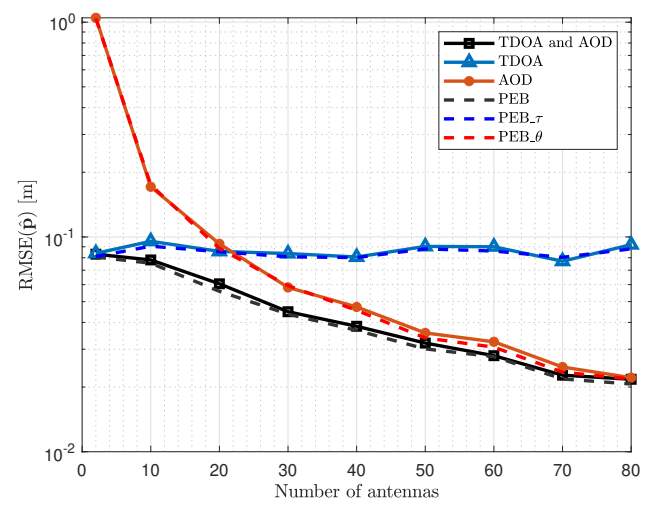

Fig. 4. RMSEs of the position estimates versus the PEB for the different estimates as a function of antennas for SNR $=5 \mathrm{~dB}$ and $W_{\mathrm{B}}=100 \mathrm{MHz}$.

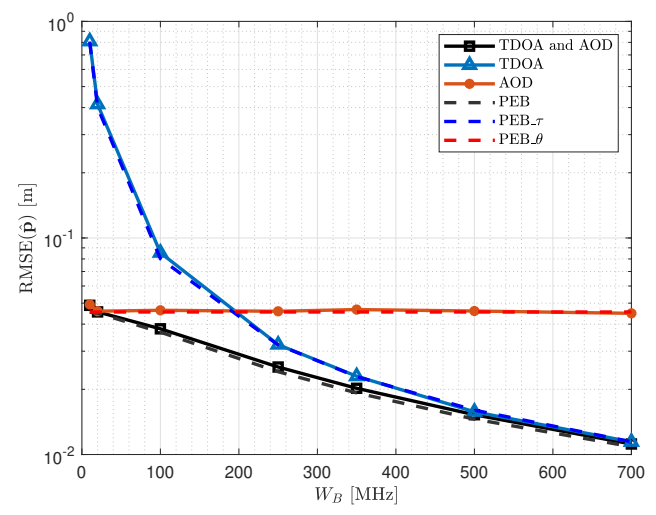

Fig. 5. RMSEs of the position estimates versus the PEB for the different estimates as a function of bandwidth for SNR $=5 \mathrm{~dB}$ and $N_{t}=40$.

\section{CONCLUSIONS}

The problem of determining the unknown MS position in a mmWave MISO system in presence of a synchronization bias is investigated. The localization process is based on the combined use of AODs and TDOAs measurements, which are estimated from pilot signals broadcast in DL by multiple BSs. We carried out a Fisher information analysis to derive the lower bounds on the estimation performance, which demonstrated the possibility to achieve sub-meter positioning accuracy. Based on such findings, we proposed a novel two-step algorithm: in the first step, the channel position-related parameters (TOAs and AODs) are estimated through a ML approach. Then, TDOAs are computed to compensate the clock bias and fed, together with the AODs, to a weighted LS estimator to retrieve the MS position. Simulation results showed that the proposed approach outperforms both AOD-only and TDOA-only competitors and is able to attain the theoretical bounds, also in presence of few transmit antennas and limited bandwidth.

An important extension of this work is the multipath case, for which the bound and estimator should be re-derived, accounting for the resolvability in the delay or angle domain.

\section{ACKNOWLEDGMENTS}

This work was supported in part by the Spanish Ministry of Science and Innovation through projects TEC2017-89925-R, the ICREA Academia Programme, and the Swedish Research Council (VR) under project 201803701.

\section{REFERENCES}

[1] J. A. del Peral-Rosado, R. Raulefs, J. A. López-Salcedo, and G. SecoGranados, "Survey of cellular mobile radio localization methods: From $1 \mathrm{G}$ to 5G," IEEE Communications Surveys Tutorials, vol. 20, pp. 11241148, Secondquarter 2018.

[2] R. Mardeni, P. Shahabi, and M. Riahimanesh, "Mobile station localization in wireless cellular systems using UTDOA," in International Conference on Microwave and Millimeter Wave Technology (ICMMT), vol. 5, May 2012.

[3] J. Liu and S. Feng, "Enhanced RSTD for scalable bandwidth of OTDOA positioning in 3GPP LTE," in International Conference on Localization and GNSS (ICL-GNSS), June 2013.

[4] Y. Sun, K. C. Ho, and Q. Wan, "Solution and analysis of TDOA localization of a near or distant source in closed form," IEEE Transactions on Signal Processing, vol. 67, pp. 320-335, Jan 2019.

[5] J. J. Caffery and G. L. Stuber, "Overview of radiolocation in CDMA cellular systems," IEEE Communications Magazine, vol. 36, pp. 38-45, April 1998.

[6] K. Cheung, H. So, W.-K. Ma, and Y. Chan, "A constrained least squares approach to mobile positioning: Algorithms and optimality," EURASIP Journal on Advances in Signal Processing, vol. 2006, Dec 2006.

[7] S. Kong and B. Kim, "Error analysis of the OTDOA from the resolved first arrival path in LTE," IEEE Transactions on Wireless Communications, vol. 15, pp. 6598-6610, Oct 2016

[8] F. Gustafsson and F. Gunnarsson, "Mobile positioning using wireless networks: possibilities and fundamental limitations based on available wireless network measurements," IEEE Signal Processing Magazine, vol. 22, pp. 41-53, July 2005.

[9] Li Cong and Weihua Zhuang, "Hybrid TDOA/AOA mobile user location for wideband CDMA cellular systems," IEEE Transactions on Wireless Communications, vol. 1, pp. 439-447, July 2002.

[10] N. J. Thomas, D. G. M. Cruickshank, and D. I. Laurenson, "Performance of a TDOA-AOA hybrid mobile location system," in Second International Conference on $3 G$ Mobile Communication Technologies, pp. 216-220, March 2001.

[11] Z. Abu-Shaban, X. Zhou, T. Abhayapala, G. Seco-Granados, and H. Wymeersch, "Error bounds for uplink and downlink 3D localization in 5G millimeter wave systems," IEEE Transactions on Wireless Communications, vol. 17, pp. 4939-4954, Aug 2018.

[12] A. Shahmansoori, G. E. Garcia, G. Destino, G. Seco-Granados, and H. Wymeersch, "Position and orientation estimation through millimeterwave mimo in 5G systems," IEEE Transactions on Wireless Communications, vol. 17, pp. 1822-1835, March 2018.

[13] A. Fascista, A. Coluccia, H. Wymeersch, and G. Seco-Granados, "Millimeter-wave downlink positioning with a single-antenna receiver," IEEE Transactions on Wireless Communications, vol. 18, no. 9, pp. 44794490, 2019.

[14] S. M. Kay, Fundamentals of Statistical Signal Processing: Estimation Theory. Prentice Hall, 1997.

[15] F. Wen, N. Garcia, J. Kulmer, K. Witrisal, and H. Wymeersch, "Tensor decomposition based beamspace esprit for millimeter wave mimo channel estimation," in IEEE Global Communications Conference (GLOBECOM), 2018. 